\title{
1 Quenched Disorder Distributions in Three-Dimensional Diluted Ferromagnets
}

\author{
W. Janke ${ }^{1}$, P.-E. Berche ${ }^{2}$, C. Chatelain ${ }^{3}$ and B. Berche ${ }^{3}$ \\ 1 Institut für Theoretische Physik, Universität Leipzig, Augustusplatz 10/11, \\ D-04109 Leipzig, Germany \\ wolfhard.janke@itp.uni-leipzig.de \\ 2 Groupe de Physique des Matériaux, Université de Rouen, F-76801 \\ Saint-Etienne du Rouvray Cedex, France \\ pierre.berche@univ-rouen.fr \\ 3 Laboratoire de Physique des Matériaux, Université Henri Poincaré, Nancy I, \\ BP 239, F-54506 Vandœuvre les Nancy Cedex, France \\ chatelai, berche@lpm.u-nancy.fr
}

Summary. We report on large-scale Monte Carlo simulations of the three-dimensional 4-state Potts ferromagnet subject to quenched, random bond dilution. For small dilutions the rather strong first-order phase transition of the pure system is found to persist, whereas for larger dilutions the theoretically expected softening to a continuous transition is confirmed. The properties of the underlying disorder distributions of thermal observables are discussed and illustrated with a few selected examples.

\subsection{Introduction}

Physical systems with quenched, random impurities display under certain conditions a completely different behaviour than the pure systems. One (often unwanted) experimental realization are point defects which may be modelled theoretically by random site dilution [1]. If the pure system exhibits a continuous phase transition, the influence of quenched, random disorder may drive the system into a new universality class, provided the critical exponent $\alpha_{\mathrm{p}}$ of the pure system's specific heat is positive (Harris criterion) [2]. Also in the case of a first-order phase transition in the pure system, quenched disorder can have a dramatic effect: Phenomenological renormalization group arguments suggest the possibility of a softening to a continuous transition [3]. While in two dimensions this effect has been theoretically proven [4] and numerically confirmed [5] for any small amount of disorder, in three dimensions (3D) one expects that with increasing disorder the strength of the transition is gradually weakened until beyond a (tricritical) concentration of impurities a real softening to a second-order transition sets in $[6,7]$.

Recent Monte Carlo (MC) computer simulations of the 3D site-diluted 3-state [8] and bond-diluted 4-state [9] Potts models, which both exhibit first-order phase transitions in the pure case, have confirmed this expectation numerically. For a complementary study using high-temperature series 

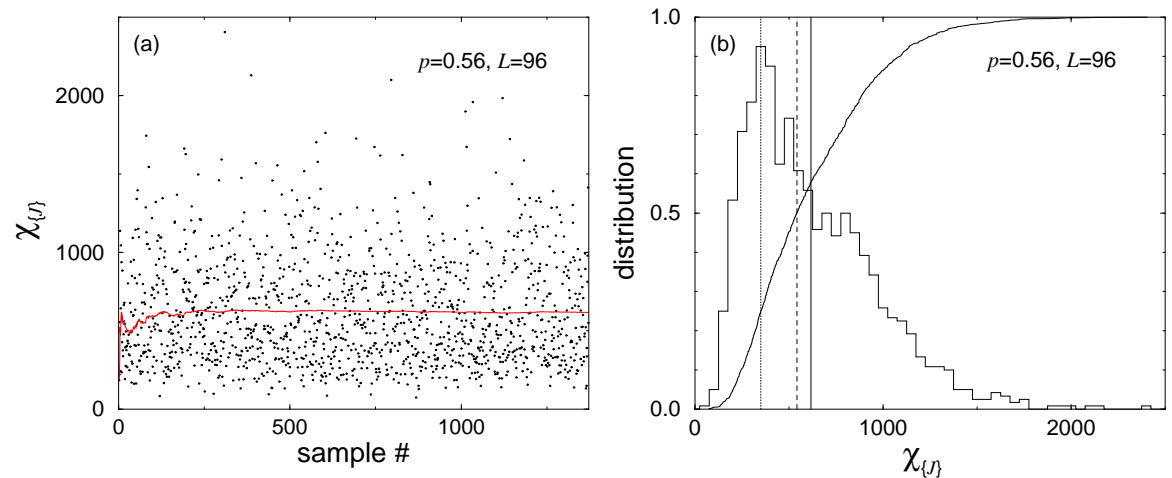

Fig. 1.1. Distribution of the magnetic susceptibility $\chi_{\{J\}}$ for $p=0.56$ and $L=96$, as obtained at a temperature close to the point where the average $\left[\chi_{\{J\}}(T)\right]_{\text {av }}$ has its maximum. The solid line in (a) is the running average, and the vertical lines in (b) show from left to right the most probable, median, and mean value.

expansions, see Ref. [10]. One important issue in MC studies is the number of independent disorder realizations that are required for reliable quenched averages. An answer to this question can usually only be given a posteriori, after having already estimated the associated distributions of thermodynamic quantities such as the susceptibility, since these distributions can be highly asymmetric. As is illustrated in Fig. 1.1, one result of our large-scale simulations [9] is that this asymmetry is particularly pronounced in the softening regime. In what follows we shall further illustrate and elucidate this effect and its consequences with a few selected examples.

\subsection{Model and Simulation Setup}

The 3D bond-diluted $q$-state Potts model is defined by the Hamiltonian

$$
H=-\sum_{\langle i j\rangle} J_{i j} \delta_{\sigma_{i}, \sigma_{j}} ; \quad \sigma_{i}=1, \ldots, q,
$$

where the sum extends over all pairs of neighbouring sites on a cubic lattice of size $L^{3}$ with periodic boundary conditions, and the couplings $J_{i j}$ are chosen according to the distribution

$$
\wp\left(J_{i j}\right)=p \delta\left(J_{i j}-J\right)+(1-p) \delta\left(J_{i j}\right),
$$

where $p$ is the concentration of magnetic bonds such that $p=1$ corresponds to the pure case where the 4-state model exhibits a rather strong first-order transition at $K_{c}(1) \equiv K_{c}=J /\left(k_{B} T_{c}\right)=0.62863(2)$.

We simulated the diluted model in the regime $p_{c}<p<p_{t}$ of second-order transitions, where $p_{c}=0.2488126(5)$ is the bond-percolation threshold [11] 


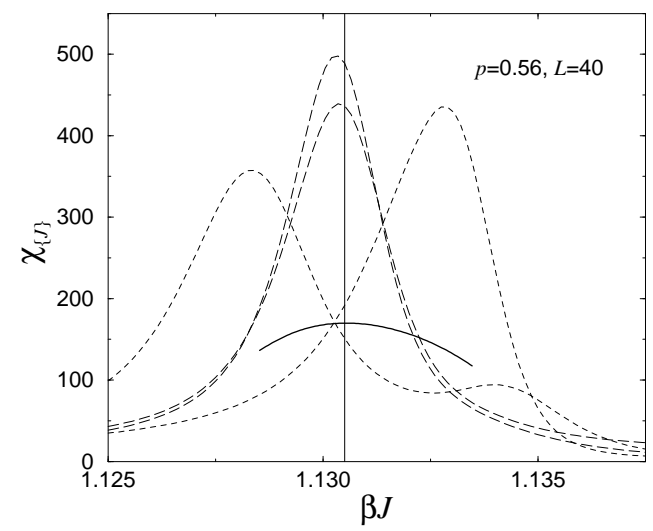

Fig. 1.2. Temperature variation of four different realizations at $p=0.56$ and $L=40$ (long and short dashed lines). The solid line with a maximum around $\chi_{\{J\}} \approx 160$ shows the average susceptibility $\chi=\left[\chi_{\{J\}}\right]_{\mathrm{av}}$.

and $p_{t} \approx 0.80$ denotes the tricritical concentration [9], with the SwendsenWang cluster algorithm [12], and in the regime $p_{t}<p \leq 1$ of first-order transitions with the multibondic method [13]. The numerically determined phase diagram in the dilution-temperature plane was found to agree very well with the single-bond effective-medium (EM) approximation [14],

$$
K_{c}^{\mathrm{EM}}(p)=\ln \left[\frac{\left(1-p_{c}\right) e^{K_{c}(1)}-(1-p)}{p-p_{c}}\right],
$$

where $K_{c}(1)$ is the transition point of the pure system and $p_{c}$ the percolation threshold given above. Our analysis of both the autocorrelation time and the interface tension [9] led to the conclusion of a tricritical point around $p=0.80$.

To arrive at these results, for each dilution, temperature and lattice size, the MC estimates $\left\langle Q_{\{J\}}\right\rangle$ of thermodynamic quantities $Q_{\{J\}}$ for a given random distribution $\{J\}$ of diluted bonds were averaged over $2000-5000$ disorder realizations,

$$
Q=\left[\left\langle Q_{\{J\}}\right\rangle\right]_{\mathrm{av}}=\int \mathcal{D} J_{i j} \wp\left(J_{i j}\right)\left\langle Q_{\{J\}}\right\rangle=\int d\left\langle Q_{\{J\}}\right\rangle \mathcal{P}\left(\left\langle Q_{\{J\}}\right\rangle\right)\left\langle Q_{\{J\}}\right\rangle,
$$

where $\mathcal{P}\left(\left\langle Q_{\{J\}}\right\rangle\right)$ denotes the empirically determined distribution of $\left\langle Q_{\{J\}}\right\rangle$ discussed in the next section.

\subsection{Distribution over Disorder Realizations}

In Fig. 1.1 the distribution of susceptibility measurements at a temperature close to the peak of its disorder average is shown for $p=0.56$ and $L=96$, i.e., 

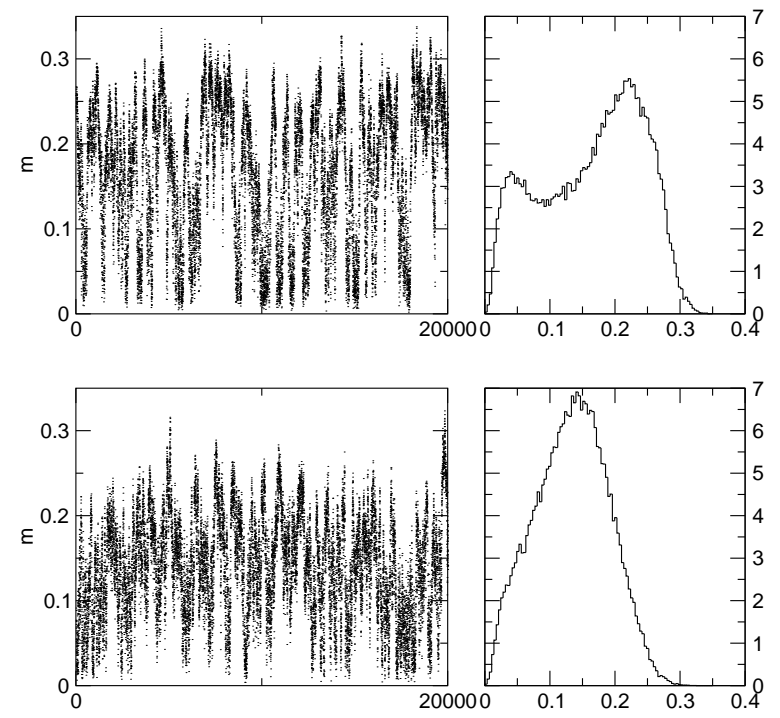

Fig. 1.3. Time evolution of the magnetization for measurements close to the (average) susceptibility peak for two different realizations at $p=0.56$ and $L=40$, and the associated thermal equilibrium distributions.

for a dilution in the centre of the softening regime $0.25 \approx p_{c}<p<p_{t} \approx 0.80$. The solid line Fig. 1.1(a) is the running average which, despite the quite asymmetric distribution, appears rather stable from about 400 realizations on. The resulting probability density (histogram) and distribution (accumulated density) are depicted in Fig. 1.1(b). By the vertical solid and dashed line we have also indicated the average and median value, respectively. The dotted vertical line at about $\chi_{\{J\}}=350$, which perfectly coincides with the most probable value, has been computed by averaging over only those $50 \%$ of the realizations whose $\chi_{\{J\}}$-value is smaller than the median value.

In Fig. 1.2 we illustrate how the average susceptibility emerges from the individual contributions of each disorder realization. The location of its maximum is indicated by the vertical line. Two events corresponding to large values of $\chi_{\{J\}}$ (rare events) are shown as long dashed lines, and two events with small values of $\chi_{\{J\}}$ (typical events) are shown as short dashed lines.

Since each $\chi_{\{J\}}$ reflects the thermal fluctuations in the magnetization $m$, we also had a closer look at the time evolution of $m$ and the associated equilibrium distribution. Two examples are shown in Fig. 1.3, where the upper part corresponds to a rare event with large susceptibility and the lower one to a typical event with smaller magnetization fluctuations. The first realization still behaves first-order like as can be seen more clearly on the right part of the figure, where the resulting thermal probability density of the magnetization exhibits a double peak. 


\subsection{Conclusions}

By performing large-scale Monte Carlo simulations we have investigated the influence of bond dilution on the thermodynamic properties of the 3D 4-state Potts model. For strong dilution with $p<0.80$ we obtained clear evidence for softening to a continuous transition. Here we have focused on a rather detailed study of the thermalization properties of individual realizations and the disorder distribution of thermodynamic quantities such as the susceptibility. Among other observations this reveals that in the softening regime a few rare realizations may still exhibit signals reminiscent of a first-order transition, e.g., a double peak in the magnetization histogram. On the one hand, this gives rise to rare events with large susceptibilities leading to quite asymmetric distributions over the disorder, and on the other hand this complicates the technical issue of deciding which Monte Carlo update algorithm is best suited for the problem at hand.

\section{Acknowledgements}

We are grateful to Meik Hellmund and Loic Turban for helpful discussions. Work partially supported by a PROCOPE collaborative grant of DAAD and EGIDE, the EU network "EUROGRID: Discrete Random Geometries: From

Solid State Physics to Quantum Gravity", the German-Israel-Foundation (GIF) grant No. I-653-181.14/1999, and the computer-time grants 2000007 of CRIHAN, hlz061 of NIC Jülich, and h0611 of LRZ München.

\section{References}

1. R. Folk, Y. Holovatch, T. Yavors'kii: Physics Uspiekhi 173, 175 (2003) [e-print cond-mat/0106468]

2. A.B. Harris: J. Phys. C 7, 1671 (1974)

3. Y. Imry, M. Wortis: Phys. Rev. B 19, 3580 (1979)

4. M. Aizenman, J. Wehr: Phys. Rev. Lett. 62, 2503 (1989)

5. B. Berche, C. Chatelain: e-print cond-mat/0207421

6. J. Cardy, J.L. Jacobsen: Phys. Rev. Lett. 79, 4063 (1997)

7. For a review, see J. Cardy: Physica A 263, 215 (1999)

8. H.G. Ballesteros, L.A. Fernández, V. Martín-Mayor, A. Muñoz Sudupe, G. Parisi, J.J. Ruiz-Lorenzo: Phys. Rev. B 61, 3215 (2000)

9. C. Chatelain, B. Berche, W. Janke, P.-E. Berche: Phys. Rev. E 64, 036120 (2001); C. Chatelain, P.-E. Berche, B. Berche, W. Janke: Nucl. Phys. B (Proc. Suppl.) 106\&107, 899 (2002); Comp. Phys. Comm. 147, 431 (2002)

10. M. Hellmund, W. Janke: Nucl. Phys. B (Proc. Suppl.) 106\&107, 923 (2002); Phys. Rev. E 67, 026118 (2003)

11. C.D. Lorenz, R.M. Ziff: Phys. Rev. E 57, 230 (1998)

12. R.H. Swendsen, J.S. Wang: Phys. Rev. Lett. 58, 86 (1987)

13. W. Janke, S. Kappler: Phys. Rev. Lett. 74, 212 (1995)

14. L. Turban: Phys. Lett. A 75, 307 (1980); J. Phys. C 13, L13 (1980) 